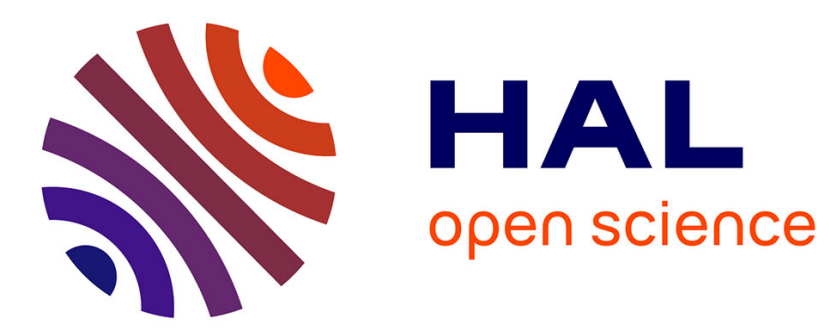

\title{
A Class of Hybrid Velocity Observers for Angular Measurements With Jumps
}

Mirko Brentari, Paolo Bosetti, Luca Zaccarian

\section{To cite this version:}

Mirko Brentari, Paolo Bosetti, Luca Zaccarian. A Class of Hybrid Velocity Observers for Angular Measurements With Jumps. IEEE Control Systems Letters, 2018, 2 (4), pp.617 - 622. 10.1109/LCSYS.2018.2845307 . hal-01851131

\section{HAL Id: hal-01851131 https://hal.laas.fr/hal-01851131}

Submitted on 10 Aug 2018

HAL is a multi-disciplinary open access archive for the deposit and dissemination of scientific research documents, whether they are published or not. The documents may come from teaching and research institutions in France or abroad, or from public or private research centers.
L'archive ouverte pluridisciplinaire HAL, est destinée au dépôt et à la diffusion de documents scientifiques de niveau recherche, publiés ou non, émanant des établissements d'enseignement et de recherche français ou étrangers, des laboratoires publics ou privés. 


\title{
A class of hybrid velocity observers for angular measurements with jumps
}

\author{
Mirko Brentari, Paolo Bosetti, and Luca Zaccarian
}

\begin{abstract}
We propose a hybrid nonlinear high-gain observer to estimate the speed of rotary systems equipped with potentiometerbased, capacitive or hall-effect rotary sensors or providing angular measurements evolving in $\mathbb{S}_{1}$, exhibiting unpredictable jumps of $2 \pi$. A hybrid measurement model is proposed, based on which a hybrid high-gain observer is synthesized, which does not require the knowledge of the jump times. Asymptotic tracking of the proposed observer is proven. A sampled-data approximation of the proposed observer is developed as well, based on which an experimental validation shows suitability for real-time applications.
\end{abstract}

\section{INTRODUCTION}

Observers are fundamental building blocks in control when facing output feedback problems. Many strategies have been proposed in order to estimate the plant state starting from its output, such as Luenberger observers [4], passivity-based observers [2], and high-gain observers [14]. Among these, high-gain observers are a well established tool when the process to be observed contains some unmodelled dynamics. They are also used in order to estimate the time derivative of the plant's output, making use of the high-gain scaling parameter as a tuning parameter allowing one to trade off between responsiveness and noise sensitivity of the observer [17], [8]. Recently, the hybrid dynamical system framework of [12] has been exploited in order to enhance the performance or extend the use of classical continuous-time observers, both in the high-gain scheme [5], [10], and in cases where hybrid dynamical system are at stake (see [7] and references therein).

Systems that evolve on the unit circle are well studied under different filtering and synchronization points of view. See the filtering problem analyzed in [9], [23], and [13], the consensus problem studied in [18], the optimization problem faced in [21], or the filtering problem on the more general special orthogonal group $\mathrm{SO}(3)$ considered in [15].

In this paper, we address state estimation for a rotational displacement process whose position evolves on $\mathbb{S}_{1}$ starting from angular measurements that evolve in the compact set $\left[0^{\circ}, 360^{\circ}\right]$, whose model is developed in Section II. In technological applications, many rotary displacement sensors exist exhibiting this feature, such as potentiometric, capacitive, or halleffect based rotational sensors. For example, [16] proposes the use of a low cost hall-effect rotary displacement sensor for controlling a synchronous reluctance machine, while [3] develops a new capacitive sensor capable of both measuring translational and rotational displacements, the latter one evolving on $\mathbb{S}_{1}$. In [1], [11], and [20], hall-effect sensors are exploited for either estimating or controlling the rotor position of permanent-magnet motors, while in [22], a capacitive angular speed sensor is proposed, along with a technological approach for estimating the rotational velocity from measurements affected by unpredictable $2 \pi$ jumps.

Our solution adopts the hybrid formalism of [12] in order to synthesize a class of hybrid high-gain observers with nonlinear injection, that estimates the speed (and a filtered version of the angular position) of a rotational displacement system evolving on the unit circle. An important feature of the proposed approach is that it is independent of the jump times. Both a hybrid continuous-discrete solution (developed in Section III) and a sampled-data solution (presented in Section V) are presented, each of them with a stability guarantees. Simulation and experimental results using a hall-effect-based sensor validate the proposed designs.

Notation. We represent with $\left(x_{1}, \ldots, x_{n}\right)$ the column-stacking operation $\left[x_{1}^{\top}, \ldots, x_{n}^{\top}\right]^{\top}$, and with $\mathbb{B}_{r}(a)$ we refer to the $n$-dimensional closed ball of radius $r$ centered at $a$, that is $\mathbb{B}_{r}(a):=\left\{x \in \mathbb{R}^{n}|| x-a \mid \leq r\right\}$.

\section{HYBRID MEASUREMENTS MODEL}

Consider a rotational system, with angular position $x_{1}$ and angular velocity $x_{2}$, following the hybrid dynamics

$$
\left\{\begin{aligned}
\dot{x} & =A x+d, & & |y| \in[0, \hat{\pi}] \\
x^{+} & =h_{1}(x), & & y \in \mathcal{J}_{1} \\
y & =C x+w=x_{1}+w, & &
\end{aligned}\right.
$$

where $x=\left(x_{1}, x_{2}\right) \in \mathcal{X}:=\left[-\frac{3}{2} \pi, \frac{3}{2} \pi\right] \times \mathbb{R}$ is the plant state and $y=x_{1}+w$ is the plant output. Moreover,

$$
\begin{aligned}
A & :=\left[\begin{array}{ll}
0 & 1 \\
0 & 0
\end{array}\right], \quad C:=\left[\begin{array}{ll}
1 & 0
\end{array}\right] \\
h_{1}(x) & :=\left[\begin{array}{c}
x_{1}-2 \pi \operatorname{sign}\left(x_{1}\right) \\
x_{2}
\end{array}\right],
\end{aligned}
$$

M. Brentari, P. Bosetti, and L. Zaccarian are with Dipartimento di Ingegneria Industriale, University of Trento, Italy. L. Zaccarian is also with LAASCNRS, Université de Toulouse, CNRS, France.

Research supported in part by grant PowerLyap funded by CaRiTRo. 


$$
\mathcal{J}_{1}:=\left[-\frac{3}{2} \pi,-\hat{\pi}\right] \cup\left[\hat{\pi}, \frac{3}{2} \pi\right],
$$

$w \in[-\rho, \rho]$ represents bounded measurement noise, $\hat{\pi}:=\pi+\delta_{\pi}$ with $\delta_{\pi}>0$, and $d \in \mathbb{R}^{2}$ is an unknown process disturbance, which may arise from unmodelled dynamics. In particular, the data of the hybrid measurement model encodes the integrating structure of the rotational system and the $2 \pi$ jump mechanism affecting the measurement. The (typically small) positive scalar $\delta_{\pi}$ prevents persistent ineffective jumps of $2 \pi$ (Zeno solutions) and the specific selection of $\delta_{\pi}>0$ does not affect the velocity estimation because it simply produces $2 \pi$ shifts of the angular estimate. Similarly, selecting $\mathcal{X}=\left[-\frac{3}{2} \pi, \frac{3}{2} \pi\right]$ enables measurement jumps in a larger set than just $[-\pi, \pi]$ so that measurement noise cannot push the state outside flow and jump sets thereby prematurely terminating solutions.

We assume that in the nominal case, i.e., $w=0$ and $d=0$, the hybrid measurement model in (1) is such that its state $x$ evolves in a compact set $\mathcal{K} \subset \mathcal{X}$, that is at constant velocity $x_{2}$ ranging in a compact set $\mathcal{K}_{2}$ as follows:

$$
x \in \mathcal{K}:=\left[-\frac{3}{2} \pi, \frac{3}{2} \pi\right] \times \mathcal{K}_{2} \subset \mathcal{X} .
$$

In the next section we propose an observer estimating $x_{2}$ without knowledge of the measurement jump times.

\section{A FAmily of High Gain Hybrid Observers}

\section{A. Observer Dynamics}

Consider the following hybrid high-gain observer with state $\hat{x}:=\left(\hat{x}_{1}, \hat{x}_{2}\right) \in \mathcal{X}$, input $\varphi$ and output $\hat{y}$ :

$$
\begin{aligned}
& \mathcal{H}_{\varphi}:= \begin{cases}\dot{\hat{x}}=A \hat{x}+L \varphi\left(e_{y}\right), & (\hat{y}, y) \in \widehat{\mathcal{C}} \\
\hat{x}^{+}=h_{1}(\hat{x}), & \hat{y} \in \mathcal{J}_{1} \\
\hat{x}^{+}=h_{2}(\hat{x}, y) & y-\hat{y} \in \mathcal{J}_{2} \\
\hat{y}=C \hat{x}=\hat{x}_{1}\end{cases} \\
& h_{2}(\hat{x}, y):=\left(y, \hat{x}_{2}\right) \\
& \mathcal{J}_{2}:=\left[-\pi-\delta_{\pi},-\pi+\delta_{\pi}\right] \cup\left[\pi-\delta_{\pi}, \pi+\delta_{\pi}\right] \\
& \widehat{\mathcal{C}}:=\left\{(\hat{y}, y) \mid \hat{y} \in \overline{\left[-\frac{3}{2} \pi, \frac{3}{2} \pi\right] \backslash \mathcal{J}_{1}},\right.
\end{aligned}
$$

where matrix $A$, the jump dynamics $h_{1}$, and the jump set $\mathcal{J}_{1}$ are defined in (1), $\varphi: \mathbb{R} \mapsto \mathbb{R}$ is a nonlinear output injection function depending on the output estimate error $e_{y}:=y-\hat{y}, L$ is a matrix of positive gains, and $h_{2}$ together with $\mathcal{J}_{2}$ provides faster transients when the output error is very large.

Assumption 1: The nonlinear output injection function $e_{y} \mapsto \varphi\left(e_{y}\right)$ is such that:

1) $\varphi$ is continuous in $\mathbb{B}_{\pi-\delta_{\pi}}(0)$,

2) $e_{y} \varphi\left(e_{y}\right)>0$ for all $e_{y} \in(-\pi, \pi) \backslash\{0\}$,

3) $\varphi\left(e_{y}\right)=\varphi\left(e_{y}+2 k \pi\right)$ for all $k \in\{-1,0,1\}$ and all $e_{y} \in \mathbb{B}_{\pi-\delta_{\pi}}(0)$,

4) $\left.\frac{d \varphi\left(e_{y}\right)}{d e_{y}}\right|_{e_{y}=0}=1$.

In Assumption 1, item 1 ensures continuity of the right hand side during flow; item 2 represents a sector condition needed for a stabilizing output injection, and ensures $\varphi\left(e_{y}\right)=0$ if and only if $e_{y}=0$; item 3 represents a periodicity condition so that the same output injection term is applied across jumps of $\pm 2 \pi$ of $e_{y}$. Without loss of generality, item 4 implies that locally the high gain observer (3) behaves as a classical linear high-gain observer with gain $L$ for every selection of $\varphi$. This assumption is nonrestrictive but it is useful for the tuning of gain $L$, and it simplifies the analysis and the generalization of Section V.

Remark 1: Items 1, 2, and 3 of Assumption 1 imply that $\varphi\left(e_{1}\right)=0$ if and only if $e_{1} \in\{2 k \pi, k \in\{-1,0,1\}\}$.

Matrix $L$ in (3a) is designed based on a pair of positive scalar gains $k_{1}$ and $k_{2}$ and a positive high-gain scaling factor $\epsilon$ as follows: $L:=\left(\ell_{1}, \ell_{2}\right):=\left(k_{1} / \epsilon, k_{2} / \epsilon^{2}\right)$. The selection of $k_{1}$ and $k_{2}$ has to be such that the characteristic equation $s^{2}+k_{v} s+k_{p}=0$ has roots with negative real part, and the high-gain scaling factor $\epsilon$ represents a design parameters that can be conveniently adjusted in order to obtain a trade-off between smoothing action (larger $\epsilon$ ) and reduction of the time lag of the estimator (smaller $\epsilon$ ) [17]. The high-gain structure also allows rejecting process noise when $\epsilon$ is small enough.

In this paper, we are interested in studying the stability properties of the interconnection between the measurement model (1) and the observer (3) with respect to the compact attractor $\mathcal{A} \subset \mathcal{K} \times \mathcal{X}$ defined as:

$$
\mathcal{A}:=\left\{(x, \hat{x}) \mid x \in \mathcal{K}, \hat{x}=x+\left[\begin{array}{c}
2 k \pi \\
0
\end{array}\right], k \in\{-1,0,1\}\right\}
$$

based on Assumption 1. The particular selection of $\mathcal{A}$ encodes the fact that we are interested in having an estimate of the position modulo $2 \pi$ rotations. The following is our main result, whose proof is reported in Section III-D.

Theorem 1: Under Assumption 1, attractor $\mathcal{A}$ in (4) is uniformly globally asymptotically stable (UGAS) for the interconnection dynamics between system (1) and (3). 


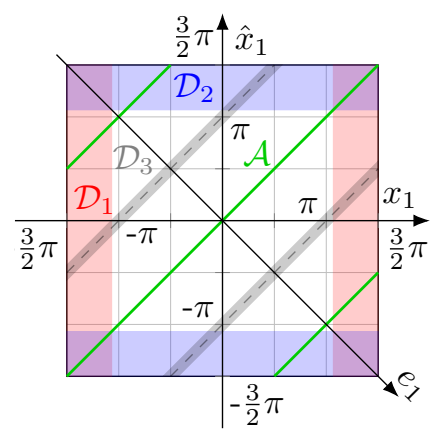

Fig. 1. Projection of the jump sets and the attractor on the $\left(x_{1}, \hat{x}_{1}\right)$ plane.

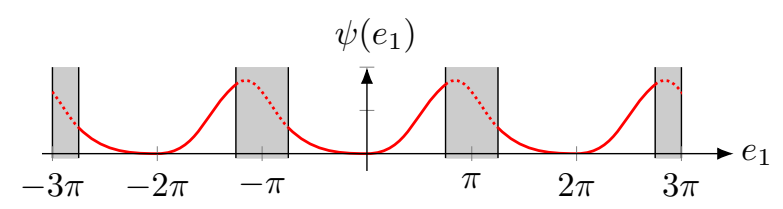

Fig. 2. Prototype of the potential function $\psi\left(e_{1}\right)$.

\section{B. The interconnected system}

To the end of proving Theorem 1, we start the analysis of the interconnection between the measurement model (1) and the observer (3) by defining the estimation error $e$ as the mismatch between the measurement model state $x$ and the estimated state $\hat{x}$ :

$$
e:=\left(e_{1}, e_{2}\right):=x-\hat{x} \in \mathcal{E}:=[-3 \pi, 3 \pi] \times \mathbb{R} .
$$

Since an autonomous representation of the tracking error dynamics $e$ cannot be written, we study the stability of $\mathcal{A}$ in (4) by writing the interconnection between plant (1) with zero inputs and observer (3) in their original coordinates $(x, \hat{x}) \in \mathcal{K} \times \mathcal{X}$, corresponding to:

$$
\left\{\begin{aligned}
(\dot{x}, \dot{\hat{x}}) & =\left[\begin{array}{ll}
A & 0 \\
0 & A
\end{array}\right](x, \hat{x})+\left[\begin{array}{l}
0 \\
L
\end{array}\right] \varphi\left(e_{1}\right) & & (x, \hat{x}) \in \mathcal{C} \\
\left(x^{+}, \hat{x}^{+}\right) & \in G(x, \hat{x}) & & (x, \hat{x}) \in \mathcal{D}
\end{aligned}\right.
$$

where

$$
\begin{aligned}
\mathcal{D} & :=\bigcup_{j \in\{1,2,3\}} \mathcal{D}_{j}, \quad \mathcal{C}:=\overline{\mathcal{K} \times \mathcal{X} \backslash \mathcal{D}} \\
\mathcal{D}_{1} & :=\mathcal{J}_{1} \times \mathcal{K}_{2} \times \mathcal{X}, \quad \mathcal{D}_{2}:=\mathcal{K} \times \mathcal{J}_{1} \times \mathbb{R} \\
\mathcal{D}_{3} & :=\left\{(x, \hat{x}) \mid x_{1}-\hat{x}_{1} \in \mathcal{J}_{2}\right\} \\
G(x, \hat{x}) & :=\bigcup_{j \in\{1,2,3\} \mid(x, \hat{x}) \in \mathcal{D}_{j}} g_{j}(x, \hat{x}) \\
g_{1}(x, \hat{x}) & :=\left(h_{1}(x), \hat{x}\right), \quad g_{2}(x, \hat{x}):=\left(x, h_{1}(\hat{x})\right) \\
g_{3}(x, \hat{x}) & :=\left(x, h_{2}\left(\hat{x}, x_{1}\right)\right) .
\end{aligned}
$$

In (6), by construction, $G$ is a set-valued mapping (multiple jumping conditions may be active at the same time) that possesses the useful property of having a closed graph because its graph is the union of the (closed) graphs of $g_{i}$. Since the elements of the jump set $\mathcal{D}$ impose jump conditions only based on $x_{1}$ and $\hat{x}_{1}$, in Figure 1 we have projected these elements on the $\left(x_{1}, \hat{x}_{1}\right)$ plane, in order to provide a graphical representation and intuition of the shape of these sets, together with the attractor $\mathcal{A}$.

\section{Lyapunov function and its properties}

Consider the following differentiable function:

$$
\psi\left(e_{1}\right):= \begin{cases}\int_{-2 \pi}^{e_{1}} \varphi(v) d v, & \text { if } e_{1} \in \mathbb{B}_{\pi-\delta_{\pi}}(-2 \pi) \\ \int_{0}^{e_{1}} \varphi(v) d v, & \text { if } e_{1} \in \mathbb{B}_{\pi-\delta_{\pi}}(0) \\ \int_{2 \pi}^{e_{1}} \varphi(v) d v, & \text { if } e_{1} \in \mathbb{B}_{\pi-\delta_{\pi}}(2 \pi) \\ \psi_{p}\left(e_{1}\right), & \text { otherwise }\end{cases}
$$


where $\psi_{p}:[-3 \pi, 3 \pi] \backslash\left\{\mathbb{B}_{\pi-\delta_{\pi}}(-2 \pi) \cup \mathbb{B}_{\pi-\delta_{\pi}}(0) \cup \mathbb{B}_{\pi-\delta_{\pi}}(2 \pi)\right\} \mapsto \mathbb{R}_{>0}$ represents a positive smooth continuation [6, Lem. 1.4.2] of the three selections of $\psi$ in the complement of the three disjoint sets at the right of (7). A prototype of $\psi$ is depicted in Figure 2. Note that from items 2 and 3 of Assumption $1, \psi$ is positive definite with respect to the set $\{-2 \pi, 0,2 \pi\}$.

To study the stability properties of the compact attractor $\mathcal{A}$ in (4) for the interconnected system (6), we construct a Lyapunov function $V: \mathcal{K} \times \mathcal{X} \mapsto \mathbb{R}$ composed by a "potential" term represented by the function $\psi$ in equation (7) depending on the position error $e_{1}$, and a "kinetic" term proportional to the square of the speed error $e_{2}$ as per (5):

$$
V(x, \hat{x}):=\psi\left(x_{1}-\hat{x}_{1}\right)+\frac{\left(x_{2}-\hat{x}_{2}\right)^{2}}{2 \ell_{2}}=\psi\left(e_{1}\right)+\frac{e_{2}^{2}}{2 \ell_{2}},
$$

Lemma 1: Under Assumption 1, given $V$ in (8) and considering the interconnected system (6), it holds that:

$$
\begin{array}{rlrl}
\dot{V}(x, \hat{x}) & =-\ell_{1} \varphi^{2}\left(e_{1}\right) \leq 0 & & \forall(x, \hat{x}) \in \mathcal{C}, \\
\Delta V(x, \hat{x}) \leq 0 & \forall(x, \hat{x}) \in \mathcal{D} .
\end{array}
$$

Proof: The gradient of $V$ with respect to $(x, \hat{x})$ is

$$
\nabla V(x, \hat{x})=\left(\varphi\left(x_{1}-\hat{x}_{1}\right), \frac{x_{2}-\hat{x}_{2}}{\ell_{2}},-\varphi\left(x_{1}-\hat{x}_{1}\right),-\frac{x_{2}-\hat{x}_{2}}{\ell_{2}}\right)
$$

and then, after scalar product with the flow dynamics (6),

$$
\begin{aligned}
\dot{V}(x, \hat{x}) & =\langle\nabla V(x, \hat{x}),(\dot{x}, \dot{\hat{x}})\rangle=-\ell_{1} \varphi^{2}\left(x_{1}-\hat{x}_{1}\right) \\
& =-\ell_{1} \varphi^{2}\left(e_{1}\right) \leq 0,
\end{aligned}
$$

which is zero if $e \in\left\{\left[\begin{array}{c}2 k \pi \\ s\end{array}\right] \mid k \in\{-1,0,1\}, s \in \mathbb{R}\right\}$ and negative otherwise (thanks to the observation in Remark 1).

With regard to $\Delta V(x, \hat{x})$, the jump map $G$ in (6d) is such that either $e_{1}$ experiences jumps of $\pm 2 \pi$ due to its elements $g_{1}$ and $g_{2}$ in (6e), leading to $V\left(x^{+}, \hat{x}^{+}\right)=V(x, \hat{x})$ (thanks to the $2 \pi$ periodicity of the potential term $\psi\left(e_{1}\right)$ in $(7)$ ), or $\left|e_{1}\right|>\left|e_{1}^{+}\right|=0$ due to its element $g_{3}$ in (6e), resulting in zeroing out the potential term of $V$, thus giving $V\left(x^{+}, \hat{x}^{+}\right)<V(x, \hat{x})$ because $(x, \hat{x}) \in \mathcal{D}_{3} \Rightarrow \psi\left(e_{1}\right)>0$.

\section{Proof of Theorem 1}

To the end of proving Theorem 1, consider the candidate Lyapunov function in (8). It holds that $V(x, \hat{x})=0, \forall(x, \hat{x}) \in \mathcal{A}$. Indeed, both the potential and the kinetic term zero out when $(x, \hat{x}) \in \mathcal{A}$, that is when $e_{1}=2 k \pi, k \in\{-1,0,1\}$ and $e_{2}=0$. Moreover, from item 2 of Assumption 1 it is immediate to see that $V(x, \hat{x})>0, \forall(x, \hat{x}) \in(\mathcal{K} \times \mathcal{X}) \backslash \mathcal{A}$. Furthermore, $V$ is radially unbounded. Indeed, the kinetic term tends to infinity as the distance from the attractor tends to infinity (recall that $x_{2} \in \mathcal{K}_{2}$, which is bounded), whereas the potential term has an argument evolving on a compact set, because both $x_{1}$ and $\hat{x}_{1}$ evolve in a compact set. From Lemma $1, \dot{V}(x, \hat{x})=-\ell_{1} \varphi^{2}\left(e_{1}\right) \leq 0$ and $\Delta V(x, \hat{x}) \leq 0$ for (6) under Assumption 1. Since we do not have strict decrease of the Lyapunov function in equation (8), we conclude the proof applying an invariance principle [19, Thm 1]. Consider any solution $\zeta$ to (6) such that $V(\zeta(0,0)) \neq 0$. If $\zeta(0,0) \in \mathcal{C}$, either $\varphi\left(e_{1}(0,0)\right) \neq 0$, and then $\dot{V}(\zeta(0,0))<0$ thanks to Lemma 1 , or $\varphi\left(e_{1}(0,0)\right)=0$. In the latter case, the flow dynamics of interconnection (6) leads to $\dot{e}_{1}=e_{2}$, which implies that there exists a arbitrary small time $\tau$ such that $\varphi\left(e_{1}(\tau, 0)\right) \neq 0$, leading to decreasing of the Lyapunov function. Consider now the case of $\zeta(0,0) \in \mathcal{D}$. Jumps due to $g_{3}$ in (6e) leads to decreasing of $V$, as discussed in the proof of Lemma 1. If $\zeta(0,0) \in \mathcal{D}_{1} \backslash \mathcal{D}_{2}$, either $\zeta(0,1) \in \mathcal{C}$ or $\zeta(0,1) \in \mathcal{D}_{3}$, leading in both cases to a decrease of $V$. A similar reasoning holds if $\zeta(0,0) \in \mathcal{D}_{2} \backslash \mathcal{D}_{1}$. If $\zeta(0,0) \in \mathcal{D}_{1} \cap \mathcal{D}_{2}$, a jump forces the solution to evolve in one of the above analyzed cases. Therefore, there is no complete solution $\zeta$ of (6) such that $V(\zeta(t, j))=V(\zeta(0,0)) \neq 0, \forall(t, j) \in \operatorname{dom}(\zeta)$, that is $V$ cannot be constant (and nonzero), completing the proof.

\section{Simulations}

In this section we illustrate the proposed hybrid observer with four different selections of the output injection nonlinearity $\varphi$, all satisfying Assumption 1. In particular, the following case studies are analyzed:

1) $\varphi\left(e_{y}\right)=\sin \left(e_{y}\right)$ [blue curves]

2) $\varphi\left(e_{y}\right)=2 \tan \left(\frac{e_{y}}{2}\right)$ [red curves]

3) $\varphi\left(e_{y}\right)=\operatorname{saw}\left(e_{y}\right)$ [green curves]

4) $\varphi\left(e_{y}\right)=\operatorname{sat}_{M}\left(\operatorname{saw}\left(e_{y}\right)\right)$ [purple curves]

where with saw $: \mathbb{R} \mapsto \mathbb{R}$ we refer to the following piecewise linear function (a sawtooth selection) $\operatorname{saw}(s):=\bmod (s+$ $\pi, 2 \pi)-\pi$, and with $\operatorname{sat}_{M}: \mathbb{R} \mapsto \mathbb{R}$ to the classical saturation function $\operatorname{sat}_{M}(s):=\max (-M, \min (s, M))$. The shape of the function is reported in Figure 3, which reveals similar small signal behaviors but different behaviors with larger errors. It easy to check that all the four case studies respect the four items of Assumption 1. In the following simulations, the same gains $k_{p}=5, k_{v}=6$, the same high-gain scaling factor $\epsilon$, and the same initial state $\hat{x}_{0}=(0,0)$ are used on all of the case studies. Scalar $\delta_{\pi}$ is set to $5^{\circ}$. 


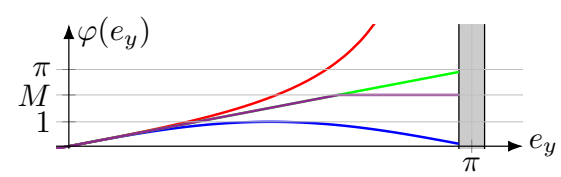

Fig. 3. The four selections of $\varphi$ proposed in Section IV.

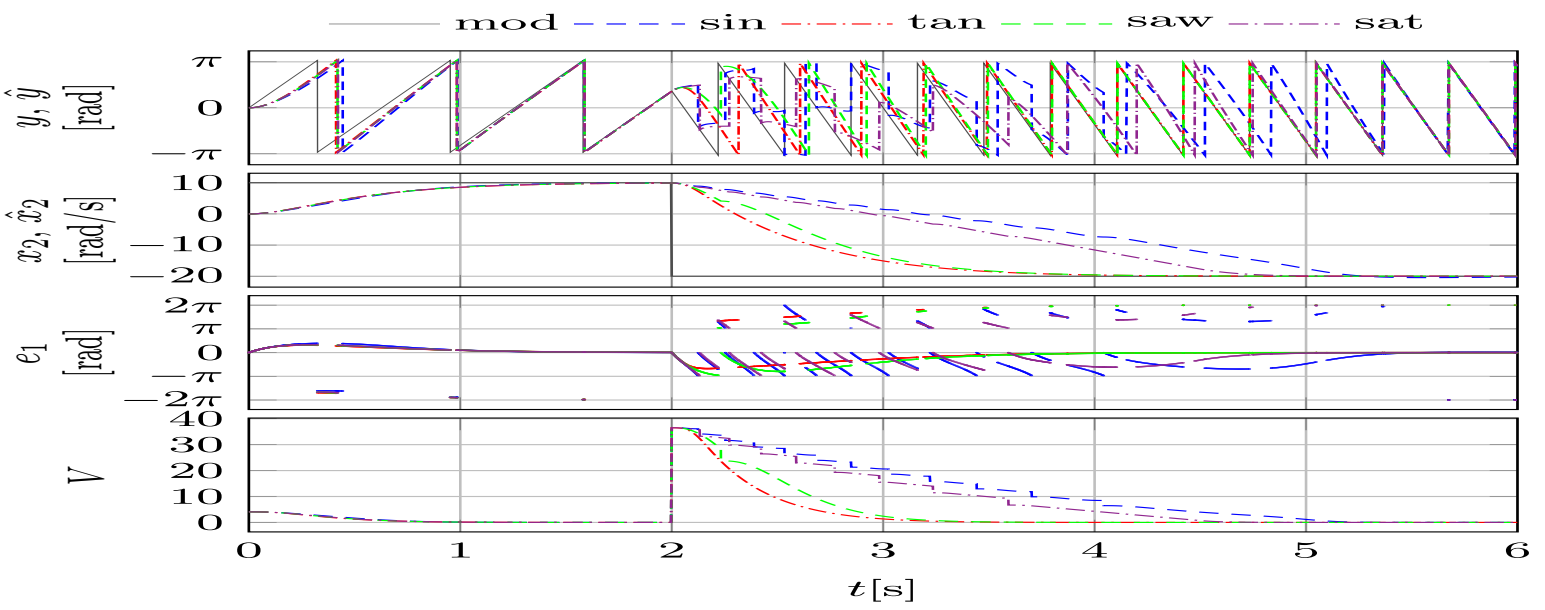

Fig. 4. Continuous-time simulation without measurement noise using the four injection functions of Section IV and $\epsilon=0.7$.

The first simulation, depicted in Figure 4, is a noise free simulation $(w=0)$, where the measurement model's initial condition is $x=(0,10)$. The initial angular velocity of $x_{2}=10 \frac{\mathrm{rad}}{\mathrm{s}}$ is sustained for the first $2 \mathrm{~s}$. Then the process disturbance $d$ quickly pushes it to $x_{2}=-20 \frac{\mathrm{rad}}{\mathrm{s}}$. In this simulation $\epsilon=0.7$ and parameter $M$ of function $\mathrm{sat}_{M}$ is 1 . It is possible to notice from the first $2 \mathrm{~s}$ of simulation that when the output tracking error $e_{y}$ is small with respect to the current selection of $\epsilon$, all the considered output-injection functions $\varphi$ produce similar responses. This is expected, since they share the same local behavior thanks to item 4 of Assumption 1 (see also Figure 3). In the second part of the simulation, the sudden variation of the angular speed $x_{2}$ produces a larger tracking error, resulting in different responses for the different selections. In particular, the best performances are the ones produced by the "tan" and "saw" output injection functions, with a slight advantage for the "tan" selection. This can be explained by the fact that the "tan" selection represents a barrier-like function with respect to the jump set $\mathcal{D}_{3}$, that is, this selection prevents the output error $e_{y}$ to become larger than $\pi-\delta_{\pi}$ in magnitude. It is not surprising that the worst response is the one produced by the "sin" and "sat" output injection functions, due to their limited action for large output errors $e_{y}$. Moreover, looking at the bottom plot of Figure 4, it is possible to observe the decrease of the Lyapunov function after jumps from $\mathcal{D}_{3}$, i.e., when $e_{1}$ approaches $\pm \pi$.

The second simulation, depicted in Figure 5, takes into account a noise signal $w \in\left[-15^{\circ}, 15^{\circ}\right]$, a scaling factor $\epsilon=0.1$ and the saturation level of function $\operatorname{sat}_{M}$ selected as $M=0.03$. Conclusions similar to those of the first simulation can be drawn.

\section{SAmpled-Data APPROXimation AND EXPERIMENTAl Results}

For real-time implementation, in this section we propose a discretized version of the observer introduced in equation (3) with periodic sampling time $T>0$.

\section{A. An equivalent sampled-data system}

Introduce a timer $\tau$ triggering the $T$-periodic sampling, and sampled versions $\bar{x}$ and $\overline{\hat{x}}$ of the measurement state $x$ and the observer state $\hat{x}$, resulting in the following extended version of dynamics $(6)$, where we denote $\xi:=(x, \hat{x}, \bar{x}, \overline{\hat{x}}, \tau)$, and where trivial jumps (namely jumps leaving the corresponding states unchanged) are omitted for conciseness:

$$
\begin{array}{rlrl}
(\dot{x}, \dot{\hat{x}}) & =\left[\begin{array}{ll}
A & 0 \\
0 & A
\end{array}\right](x, \hat{x})+\left[\begin{array}{l}
0 \\
L
\end{array}\right] \varphi\left(e_{1}\right) & & \xi \in \mathcal{C}^{\xi} \\
(\dot{\bar{x}}, \dot{\hat{x}}, \dot{\tau}) & =(0,0,1) & \\
\xi^{+} \in G^{\xi}(\xi) & \xi \in \mathcal{D}^{\xi} .
\end{array}
$$




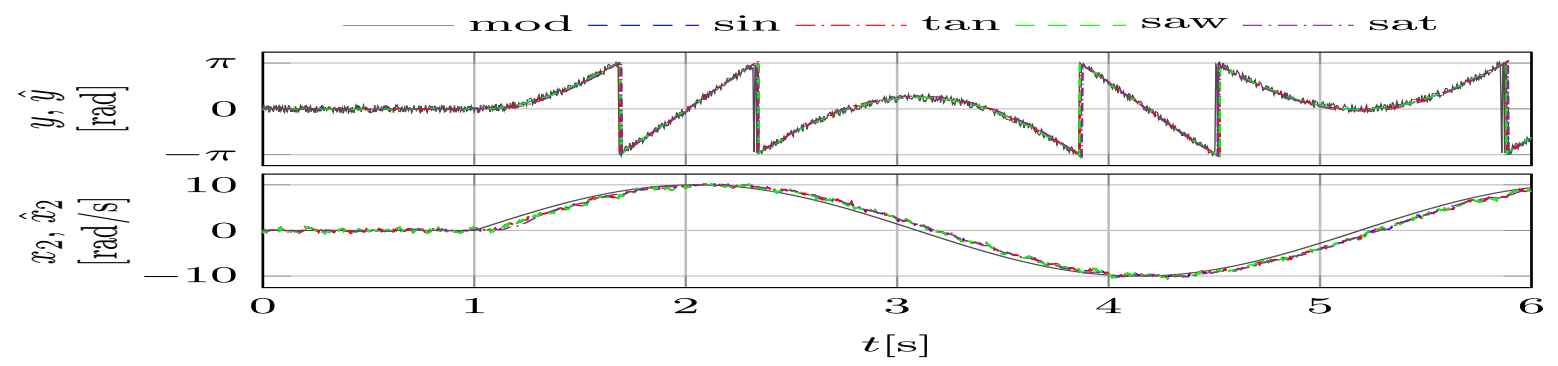

Fig. 5. Continuous-time simulation with measurement noise using the four injection functions of Section IV and $\epsilon=0.1$.

Above, the jump set is the union $\mathcal{D}^{\xi}:=\mathcal{D}_{1}^{\xi} \cup \mathcal{D}_{T}^{\xi}$, and the jump map $G^{\xi}$ is selected, similarly to (6d), as the outer semicontinuous hull $G^{\xi}(\xi):=\bigcup_{j \in\{1, T\} \mid \xi \in \mathcal{D}_{j}^{\xi}} g_{j}^{\xi}(\xi)$ of the measurement jump rule ${ }^{1}$ inherited from (6c),

$$
x^{+}=h_{1}(x), \quad \xi \in \mathcal{D}_{1}^{\xi}:=\left\{\xi: x \in \mathcal{D}_{1}\right\},
$$

and the sampling-related jump, resetting the timer to zero, implementing the jumps of $\hat{x}$ from (6c), and updating the sampled states $(\bar{x}, \overline{\hat{x}})$,

$$
\begin{aligned}
& \overline{\hat{x}}^{+}=\hat{x}^{+} \in \bar{h}_{1}\left(\bar{h}_{2}(\hat{x}, y)\right), \quad \xi \in \mathcal{D}_{T}^{\xi}:=\{\xi: \tau=T\} . \\
& (\bar{x}, \tau)^{+}=(x, 0),
\end{aligned}
$$

where $\bar{h}_{1}$ and $\bar{h}_{2}$ are the following outer semicontinuous extensions of function $h_{1}$ and $h_{2}$ in (1c) and (3b):

$$
\begin{gathered}
\bar{h}_{1}(x):= \begin{cases}h_{1}(x), & \text { if } x_{1} \in \mathcal{J}_{1} \backslash \partial \mathcal{J}_{1} \\
x, & \text { if } x_{1} \notin \mathcal{J}_{1} \\
\{x\} \cup\left\{h_{1}(x)\right\}, & \text { if } x_{1} \in \partial \mathcal{J}_{1} .\end{cases} \\
\bar{h}_{2}(\hat{x}, y):= \begin{cases}h_{2}(\hat{x}, y), & \text { if } y-\hat{x}_{1} \in \mathcal{J}_{2} \backslash \partial \mathcal{J}_{2} \\
\hat{x}, & \text { if } y-\hat{x}_{1} \notin \mathcal{J}_{2} \\
\{\hat{x}\} \cup\left\{h_{2}(\hat{x}, y)\right\}, & \text { if } y-\hat{x}_{1} \in \partial \mathcal{J}_{2} .\end{cases}
\end{gathered}
$$

The model is completed by the selection of the flow set:

$$
\mathcal{C}^{\xi}:=\left\{\xi:(x, \hat{x}) \in \overline{(\mathcal{K} \times \mathcal{X}) \backslash \mathcal{D}_{1}}, \tau \in[0, T]\right\},
$$

which enforces flowing in the interior of the sampling interval $\tau \in(0, T)$ unless the measurement model jumps (from $\mathcal{D}_{1}^{\xi}$ ). The following proposition is a key step towards our sampled-data implementation of the next section.

Proposition 1: There exists a small enough $T^{*}$ such that for any $T \in\left(0, T^{*}\right]$, the following compact set

$$
\begin{array}{r}
\mathcal{A}^{\xi}:=\{\xi: \tau \in[0, T], x-\hat{x} \in \mathcal{P}, \bar{x}-\overline{\hat{x}} \in \mathcal{P}, \\
x-(I+A \tau) \bar{x} \in \mathcal{P}\},
\end{array}
$$

(where $\mathcal{P}:=\left\{\left[\begin{array}{c}2 k \pi \\ 0\end{array}\right] \mid k \in\{-1,0,1\}\right\}$ represents the $2 \pi$ shifted origin) is UGAS for system (11).

Proof: First note that after at most $T$ ordinary time, any solution jumps from $\mathcal{D}_{T}^{\xi}$ and then evolves forever in the forward invariant manifold $\mathcal{M}:=\{\xi: x-(I+A \tau) \bar{x} \in \mathcal{P}\}$. From this manifold, since the flow dynamics of (11) coincide with those of (6) the Lyapunov flow condition established in the proof of Theorem 1 still holds. Similar to the proof of Theorem 1, jumps from both $\mathcal{D}_{1}^{\xi}$ and $\mathcal{D}_{T}^{\xi}$ cause no increase of the Lyapunov function and the result is implied by persistent flowing and the invariance principle.

\section{B. Approximated sampled-data system}

Let us consider a solution of the sampled-data model (11) and denote by $\left(t_{j}, j-1\right)$ any jump time such that $\tau\left(t_{j}, j-1\right)=T$ and $\tau\left(t_{j}, j\right)=0$. Exploiting the specific structure of matrix $A$, we have for all $t$ before the next jump,

$$
x(t, j)=(I+A \tau(t, j)) \bar{x}(t, j)=(I+A \tau(t, j)) \bar{x}\left(t_{j}, j\right) .
$$

${ }^{1}$ For compact notation, we don't define explicitly maps $g_{j}^{\xi}(\xi), j=1, T$, we only report in (11b)-(11c) the quantities that perform nontrivial jumps. 
Then we may write an autonomous version of the dynamics of $\bar{x}$ and of a sampled version $\bar{y}$ of output $y=C x$, by replacing the update of $\bar{x}$ in (11c) by

$$
\bar{x}^{+}=(I+A T) \bar{x}, \quad \bar{y}:=C \bar{x}^{+}=C(I+A T) \bar{x},
$$

where we emphasize that output $y$ at sampling times depends on the current continuous state $x$, which is the future sample $\bar{x}^{+}$of the sampled state $\bar{x}$. Inspired by (14) we propose the following sampled-data approximation of the jumps of $\overline{\hat{x}}$ in (11c)

$$
\begin{aligned}
& \overline{\hat{x}}^{+}=h_{1}\left(\bar{h}_{2}((I+A T) \overline{\hat{x}}+T L \varphi(\bar{y}-\overline{\hat{y}}), \bar{y})\right) \\
& \overline{\hat{y}}:=C(I+A T) \overline{\hat{x}}
\end{aligned}
$$

which enjoys the property in the next theorem.

Theorem 2: For any Lipschitz function $\varphi$ satisfying Assumption 1 and some bounded set of initial conditions $\overline{\hat{x}}(0,0)$, the sampled-data observer (15), (14) guarantees practical estimation of the sampled version $\bar{x}$ of state $x$ with respect to parameter $T$.

Proof: The UGAS of (12) established in Proposition 1 is semiglobally practically robust in the sense of [12, Lemma 7.20]. We may then consider a perturbed version of dynamics (11) where the upper equation in (11a) is replaced by

$$
(\dot{x}, \dot{\hat{x}})=\left[\begin{array}{ll}
A & 0 \\
0 & A
\end{array}\right](x, \hat{x})+\left[\begin{array}{l}
0 \\
L
\end{array}\right] \varphi(C(I+A T)(\bar{x}-\overline{\hat{x}})),
$$

which we denote by $((11) \leftarrow(16))$ for simplicity of notation. We may well interpret $((11) \leftarrow(16)))$ as a perturbed version of (11) where the right hand side of (11a) is affected by disturbance

$$
L d:=L(\varphi(C(I+A T)(\bar{x}-\overline{\hat{x}}))-\varphi(C(x-\hat{x}))) .
$$

For the ensuing system, intersecting the flow and jump sets with any arbitrarily large set $\left\{\hat{x}_{2} \in\left[-\hat{x}_{2 M}, \hat{x}_{2 M}\right]\right\}$, we obtain the uniform bound (where $L_{\varphi}$ represents a Lipschitz bound and $\varphi_{M}$ represents a global bound for $\varphi(\cdot)$ ):

$$
\begin{aligned}
|d| & \leq|\varphi(C(I+A T)(\bar{x}-\overline{\hat{x}}))-\varphi(C(\bar{x}-\overline{\hat{x}}))| \\
& \quad+|\varphi(C(\bar{x}-\overline{\hat{x}}))-\varphi(C(x-\hat{x}))| \\
& \leq L_{\varphi}\left(T\left(x_{2 M}+\hat{x}_{2 M}\right)+T\left|x_{1}-\bar{x}_{1}\right|+T\left|\hat{x}_{1}-\overline{\hat{x}}_{1}\right|\right),
\end{aligned}
$$

which shrinks to zero as $T$ is reduced because $\left|\dot{\hat{x}}_{1}\right| \leq \hat{x}_{2 M}+\ell_{1} \varphi_{M}$ and $\left|\dot{x}_{1}\right| \leq x_{2}$, which belongs to compact set $\mathcal{K}_{2}$. The possibility of shrinking $d$ to zero by reducing $T$ enables applying [12, Lemma 7.20] and establishing semiglobal practical UGAS of $\mathcal{A}^{\xi}$ in (12).

The proof is then completed by noticing that the term $\varphi(C(I+A T)(\bar{x}-\overline{\hat{x}}))$ forcing the right hand side of (16) is constant and equal to $\varphi(\bar{y}-\overline{\hat{y}})$. Therefore the value of $\overline{\hat{x}}^{+}$in (15) coincides with that of $\overline{\hat{x}}^{+}$in (11c). Practical convergence of $\overline{\hat{x}}$ to $\bar{x}$ then follows from practical UGAS of $\mathcal{A}^{\xi}$ in (12) and the fact that $\overline{\hat{x}}=\bar{x}$ in $\mathcal{A}^{\xi}$.

\section{Real-time implementation and experiments}

A ready-to-code algorithm implementing the sampled-data observer (15) can be found in Algorithm 1. Four realizations of Algorithm 1 have been coded in $\mathrm{C}++$ in a 32bit 120MHz ARM Cortex-M4 microprocessor. Each one of the four realization represents one of the case studies of output injection function $\varphi$ presented in Section IV. The microprocessor reads out the angular measurements via an I2C bus from an hall-effect AMS AS5048 encoder, which is an easy-to-use 14bit resolution

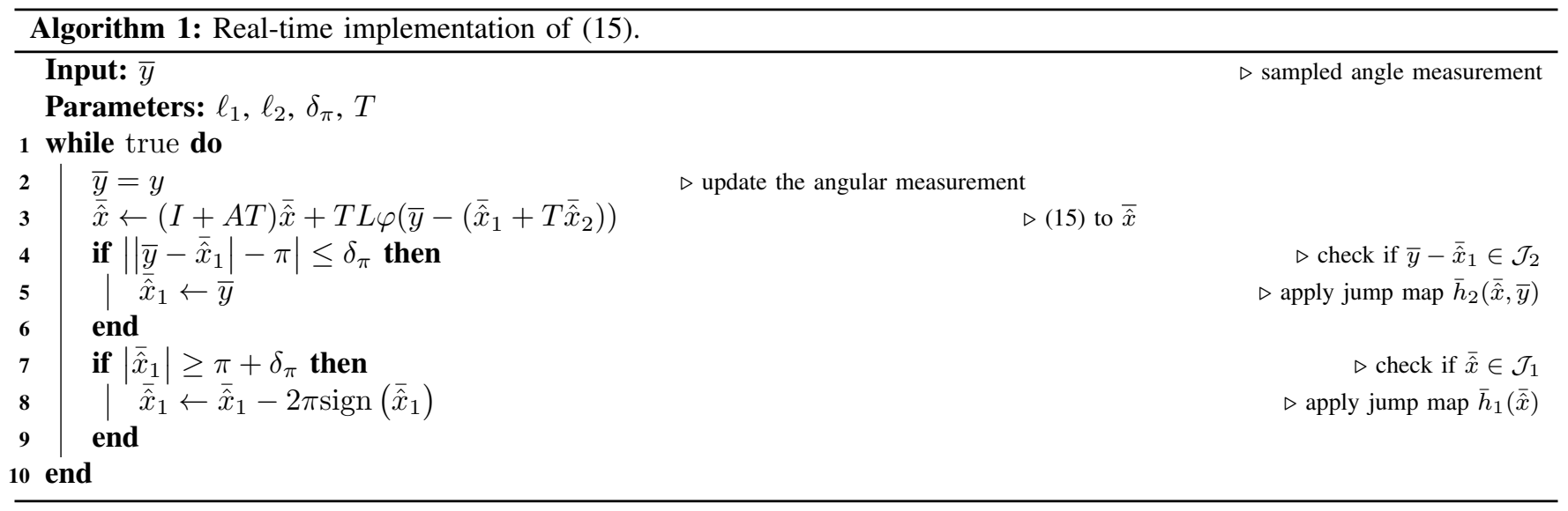



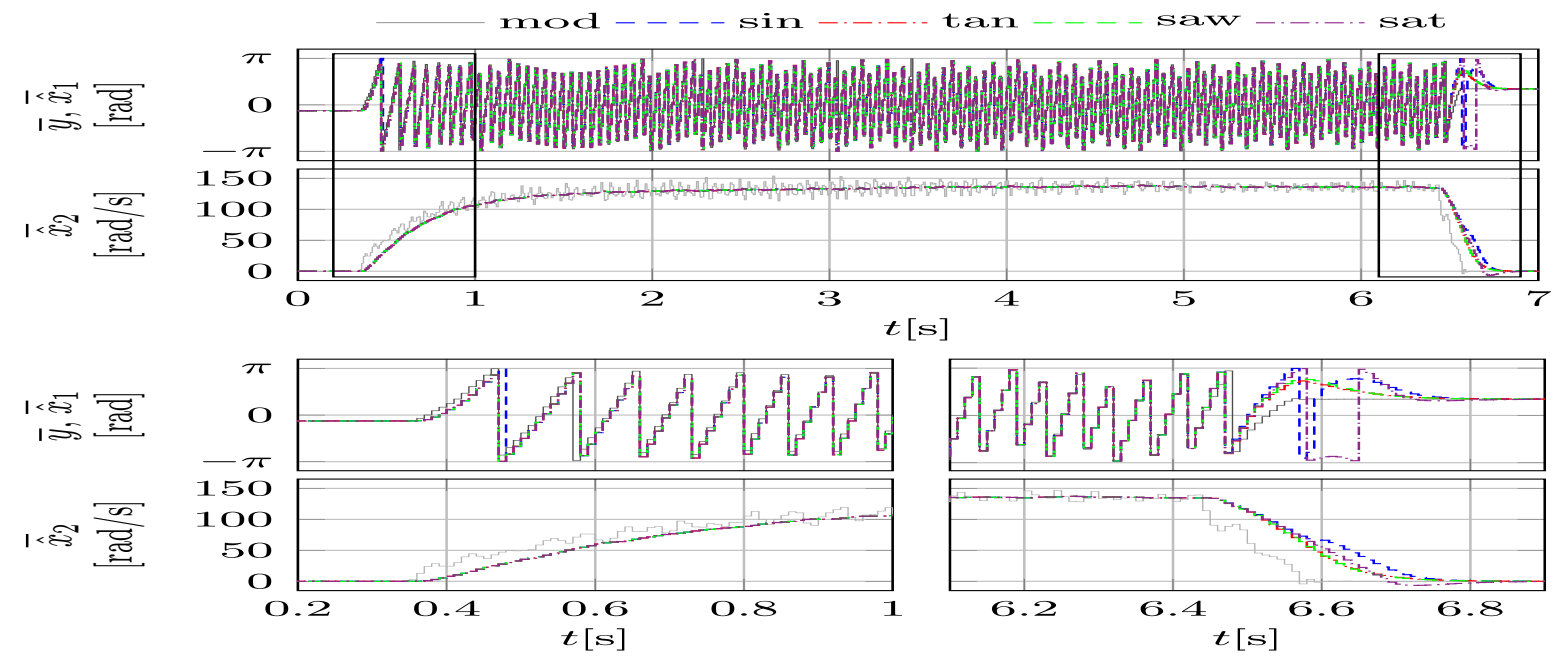

Fig. 6. Experimental results using the four injection functions. $\epsilon=0.1$.

absolute angular sensor for industrial applications. In our experiments the encoder measures the angular displacement of the shaft of a small DC brushed motor that is open-loop voltage controlled. The algorithms run in real time at $100 \mathrm{~Hz}$ and the observer parameters $k_{p}$ and $k_{v}$ are set as in the simulations to respectively 5 and 6 , while $\delta_{\pi}=5^{\circ}$, and $M=1$. The results of one of the experiments are depicted in Figure 6. In the plots relative to the angular velocity estimation we added in light gray a dirty derivative of a rectified version of the sampled measurement $\bar{y}$, which was computed off-line. The DC motor is supplied with a voltage inducing a rotation with approximate speed of $150 \frac{\mathrm{rad}}{\mathrm{s}}$. In the acceleration phase the motor reaches the steady state in about $2 \mathrm{~s}$, and the high-gain scaling factor $\epsilon$ is such that all the case studies behave similarly, confirming the simulation results. Similar behaviors are produced at the steady state as well, where the dirty derivative curve confirms that the angular velocity estimated by the real-time observer implementation is correct and well filtered. In the deceleration phase the motor slows down more promptly, making the differences among the four considered case studies more evident. The experimental results confirm that the increased aggressiveness of the "tan" and "saw" output injection functions produce a faster transient, while the limited forcing actions of the "sin" and "sat" selections produce a slower transient.

\section{Vi. Conclusions}

In this paper, we proposed a nonlinear hybrid speed observer structure for angular measurements affected by jumps. Stability properties of the proposed observer have been established with a Lyapunov-based argument under some natural assumptions on the nonlinear injection function. After illustrating some continuous-time simulation, a sampled-data approximated version of the proposed observer was developed for real time implementation. Experimental results of the discretized observer have been reported, showing that the proposed algorithm is suitable for mechatronic applications.

\section{REFERENCES}

[1] H.J. Ahn and D.M. Lee. A new bumpless rotor-flux position estimation scheme for vector-controlled washing machine. IEEE Trans. Ind. Informat., 12(2):466-473, 2016.

[2] A. Ailon and R. Ortega. An observer-based set-point controller for robot manipulators with flexible joints. Systems \& Control Letters, 21(4):329-335, 1993.

[3] N. Anandan and B. George. A wide-range capacitive sensor for linear and angular displacement measurement. IEEE Trans. Ind. Electron., 64(7):57285737, 2017.

[4] V. Andrieu and L. Praly. On the existence of a kazantzis-kravaris/luenberger observer. SIAM Journal on Control and Optimization, 45(2):432-456, 2006.

[5] V. Andrieu, C. Prieur, S. Tarbouriech, and L. Zaccarian. A hybrid scheme for reducing peaking in high-gain observers for a class of nonlinear systems. Automatica, 72:138-146, 2016.

[6] D. Barden and C. Thomas. An introduction to differential manifolds. Imperial College Press, 2003.

[7] A. Bisoffi, L. Zaccarian, M. Da Lio, D. Carnevale, and JET Contributors. Hybrid cancellation of ripple disturbances arising in AC/DC converters. Automatica, 77:344-352, 2017.

[8] Y. Chitour. Time-varying high-gain observers for numerical differentiation. IEEE Trans. Autom. Control, 47(9):1565-1569, 2002.

[9] P. Coote, J. Trumpf, R. Mahony, and J.C. Willems. Near-optimal deterministic filtering on the unit circle. In IEEE Conference on Decision and Control, pages 5490-5495, 2009.

[10] N. Cox, L. Marconi, and A.R. Teel. High-gain observers and linear output regulation for hybrid exosystems. International Journal of Robust and Nonlinear Control, 24(6):1043-1063, 2014.

[11] D. Diaz, D. Fernandez, C. Gonzales, S.B. Lee, and F. Briz. Permanent magnet synchronous machine drive control using analog hall-effect sensors. IEEE Trans. Ind. Appl., 2018.

[12] R. Goebel, R.G. Sanfelice, and A.R. Teel. Hybrid Dynamical Systems: modeling, stability, and robustness. Princeton University Press, 2012.

[13] M. F. Haydar and M. Lovera. $H_{\infty}$ filtering on the unit circle. In IEEE Conference on Decision and Control, pages 2422-2427, Dec 2017. 
[14] H. Khalil and L. Praly. High-gain observers in nonlinear feedback control. International Journal of Robust and Nonlinear Control, 24(6):993-1015, 2014.

[15] R. Mahony, T. Hamel, and J.M. Pflimlin. Nonlinear complementary filters on the special orthogonal group. IEEE Trans. Autom. Control, 53(5):1203$1218,2008$.

[16] L. Masisi and P. Pillay. High-resolution low-cost rotor position sensor for traction applications. In IEEE IECON, pages 1971-1976, Oct 2017.

[17] S. Nicosia, A. Tornambè, and P. Valigi. Experimental results in state estimation of industrial robots. In IEEE Conference on Decision and Control, pages 360-365, 1990.

[18] A. Sarlette, S. Emre Tuna, Vincent D Blondel, and R. Sepulchre. Global synchronization on the circle. IFAC Proceedings Volumes, 41(2):9045-9050, 2008.

[19] A. Seuret, C. Prieur, S. Tarbouriech, A.R. Teel, and L. Zaccarian. A nonsmooth hybrid invariance principle applied to robust event-triggered design. Submitted to IEEE Transactions on Automatic Control. Also available online at HAL 01526331, 2018.

[20] P. Stewart and V. Kadirkamanathan. Commutation of permanent-magnet synchronous ac motors for military and traction applications. IEEE Trans. Ind. Electron., 50(3):629-630, 2003.

[21] T. Strizic, J. I. Poveda, and A.R. Teel. Hybrid gradient descent for robust global optimization on the circle. In IEEE Conference on Decision and Control, pages 2985-2990, Dec 2017.

[22] F. Tibor and G. Brasseur. A robust capacitive angular speed sensor. IEEE Trans. Instrum. Meas., 47(1):280-284, 1998.

[23] M. Zamani, J. Trumpf, and R. Mahony. Minimum-energy filtering on the unit circle. In Australian Control Conf., pages 236-241, 2011. 